\title{
Sinorhizobium meliloti Functionally Replaces 3-Oxoacyl-Acyl Carrier Protein Reductase (FabG) by Overexpressing NodG During Fatty Acid Synthesis
}

\author{
Ya-Hui Mao, Feng Li, Jin-Cheng Ma, Zhe Hu, and Hai-Hong Wang \\ Guangdong Provincial Key Laboratory of Protein Function and Regulation in Agricultural Organisms, College of Life Sciences, \\ South China Agricultural University, Guangzhou, Guangdong 510642, China \\ Submitted 19 March 2015. Accepted 6 March 2016.
}

\begin{abstract}
In Sinorhizobium meliloti, the nodG gene is located in the nodFEG operon of the symbiotic plasmid. Although strong sequence similarity (53\% amino acid identities) between $S$. meliloti NodG and Escherichia coli FabG was reported in 1992, it has not been determined whether $S$. meliloti NodG plays a role in fatty acid synthesis. We report that expression of $S$. meliloti NodG restores the growth of the $E$. coli fabG temperature-sensitive mutant CL104 under nonpermissive conditions. Using in vitro assays, we demonstrated that NodG is able to catalyze the reduction of the 3-oxoacyl-ACP intermediates in $E$. coli fatty acid synthetic reaction. Moreover, although deletion of the $S$. meliloti nodG gene does not cause any growth defects, upon overexpression of nodG from a plasmid, the $S$. meliloti fabG gene encoding the canonical 3-oxoacyl-ACP reductase (OAR) can be disrupted without any effects on growth or fatty acid composition. This indicates that $S$. meliloti nodG encodes an OAR and can play a role in fatty acid synthesis when expressed at sufficiently high levels. Thus, a bacterium can simultaneously possess two or more OARs that can play a role in fatty acid synthesis. Our data also showed that, although SmnodG increases alfalfa nodulation efficiency, it is not essential for alfalfa nodulation.
\end{abstract}

3-Oxoacyl-ACP reductase (OAR), also called FabG, catalyzes the essential 3-oxoacyl-reduction step in the bacterial fatty acid synthesis pathway (Campbell and Cronan 2001; White et al. 2005; Zhang and Rock 2008). OAR is a member of a very large family of enzymes, the short-chain alcohol dehydrogenase/reductase (SDR) family, which carries out a wide range of reduction and dehydrogenation reactions using NADH or NADPH (Kavanagh et al. 2008; Oppermann et al. 2003). Although only a 15 to $30 \%$ sequence identity exists among different SDR proteins, they share a few sequence motifs, such as the GXXXGXG coenzymebinding site in the N-terminal region and the YXXXK catalytic site in the central region (Kavanagh et al. 2008; Oppermann et al. 2003). Therefore, a true OAR can be very difficult to distinguish by bioinformatic analyses from other SDR family members that catalyze unrelated reactions.

Y. H. Mao and F. Li contributed equally to this work.

Corresponding author: H.-H. Wang; Telephone: +(86) 020-85281389; Fax: +(86) 020-85282180; E-mail: wanghh36@scau.edu.cn

*The $\boldsymbol{e}$-Xtra logo stands for "electronic extra" and indicates that five supplementary figures and one supplementary table are published online.

() 2016 The American Phytopathological Society $f a b G$ is the only gene that encodes OAR (Lai and Cronan 2004) in Escherichia coli. However, in other species of bacteria, multiple FabG paralogs have been annotated. For example, the genome of Mycobacterium tuberculosis has five annotated fabG genes (Gurvitz 2009). In Pseudomonas aeruginosa, at least four genes have been annotated as encoding FabG homologs (Stover et al. 2000). However, in most cases, only one of the homologs in these bacteria was identified as the OAR that plays a role in fatty acid synthesis. Lactococcus lactis possesses two putative FabGs, but only FabG1 had OAR activity and a role in fatty acid synthesis (Wang and Cronan 2004). Thus, to date, it has been believed that only a single enzyme, FabG, catalyzes the reduction of 3-oxoacyl-ACPs to 3-hydroxyacyl-ACPs in bacterial fatty acid synthetic pathways (Zhang and Rock 2008).

Recently however, we reported the first example of a bacterium that encodes two functional OARs. Ralstonia solanacearum, a soil-borne plant pathogen, has two genes, RSc1052 (fabGl) and RSp0359 ( $f a b G 2$ ), that encode OAR. This suggested that one bacterium could simultaneously possess two or more OARs (Feng et al. 2015).

Rhizobial bacteria have the unique ability to induce nitrogenfixing nodules on the roots or stems of leguminous plants (Galibert et al. 2001). The development of legume nodules is largely controlled by reciprocal signal exchange between these symbiotic partners (Debellé et al. 2001; Mergaert et al. 1997). Plant-specific flavonoids induce the rhizobial nod genes needed for nodulation. Most of these genes are involved in the synthesis of Nod factors (lipochitin oligosaccharides) that trigger nodule formation. In all cases, the basic structure of Nod factors consists of a chitin oligosaccharide backbone and fatty acids that are $\mathrm{N}$-acylated on the nonreducing terminal residue of oligosaccharides (Debellé et al. 2001; Mergaert et al. 1997).

In Sinorhizobium meliloti, it has been demonstrated that the $\operatorname{nod} A, \operatorname{nod} B$, and nod $C$ genes are needed for the chitin oligosaccharide backbone synthesis of the Nod factors and that nodF and $n o d E$ are the only genes required for the production of unusual $\alpha, \beta$-unsaturated fatty moieties of the Nod factors (Debelle et al. 2001). The nodG gene is also located in the nodFEG operon, but mutations in the $S$. meliloti nod $G$ do not result in any detectable change in the unusual $\alpha, \beta$-unsaturated fatty acids structure of the Nod factors (Demont et al. 1993) and nodG mutants inoculated on alfalfa do not show any defect in nodulation (Swanson et al. 1987). These indicate that nodG is not required for synthesis of the unusual $\alpha, \beta$-unsaturated acyl chains and is not essential for alfalfa nodulation. Moreover, although a strong NodG sequence similarity to E. coli FabG was reported in 1992 (Rawlings and Cronan 1992), the function of $S$. meliloti NodG is still unknown. As with $S$. meliloti, Rhizobium leguminosarum also 
possesses nodG. It has been suggested that NodG, like FabG during fatty acid synthesis by Rhizobium leguminosarum, has OAR activity in vitro (López-Lara and Geiger 2001), but there has been no further evidence to confirm that Rhizobium leguminosarum NodG plays a role in fatty acid synthesis in vivo.

We report that $S$. meliloti NodG possesses OAR activity and upon overexpression of NodG from a plasmid, the $f a b G$, which encodes the canonical OAR in fatty acid synthesis, could be disrupted.

\section{RESULTS}

\section{S. meliloti nodG complemented the growth} of the $E$. coli fabG temperature-sensitive mutant strain.

Alignments of $S$. meliloti NodG with E. coli FabG and S. meliloti FabG indicated that NodG was 52.8 and $79.6 \%$ identical to E. coli FabG and S. meliloti FabG, respectively (Galibert et al. 2001), and NodG contained the SDR family catalytically active triad (Ser, Tyr, and Lys) plus the N-terminal cofactor binding sequence (Gly motif [GXXXGXG]) (Kavanagh et al. 2008; Oppermann et al. 2003). It has been demonstrated that Rhizobium leguminosarum NodG protein possessed OAR activity in vitro (López-Lara and Geiger 2001). To test the putative OAR functions of $S$. meliloti NodG in vivo, the $S$. meliloti nod $G$ and $f a b G$ genes were inserted into the pBAD24M vector, in which transcription of the foreign genes would proceed from the arabinose-regulated araBAD promoter. This manipulation resulted in plasmids pLF4 (pBADSmnodG) and pLF5 (pBAD-SmfabG), which were transformed into the E. coli fabG(Ts) strain CL104 (Lai and Cronan 2004) at $30^{\circ} \mathrm{C}$. The resulting transformants were tested for growth on rich broth $(\mathrm{RB})$ plates at the nonpermissive temperature of $42^{\circ} \mathrm{C}$. The derivatives of CL104 that carried pLF4 (pBAD-SmnodG) or pLF5 (pBAD-SmfabG) grew well at $42^{\circ} \mathrm{C}$ in the presence of arabinose. However, in the absence of arabinose, the CL104 strain that carried pLF4 (pBAD-SmnodG) grew less well than the CL104 strain that carried pLF5 (pBAD-SmfabG) (Fig. 1A). The growth of these strains in liquid $\mathrm{RB}$ medium containing arabinose was also tested (Fig. 1B). The data showed that the strain CL104 derivative that carried pLF4 (pBAD-SmnodG) had a longer lag phase before it entered logarithmic growth than the strain CL104 derivative that carried pLF5 (pBAD-SmfabG) (Fig. 1B). These results indicated that both $S$. meliloti nodG and fabG could complement the E. coli fabG(Ts) strain. However, it appears likely that $\mathrm{SmNodG}$ is less active than $\mathrm{SmFabG}$, since high-level induction by arabinose was required for robust growth.

\section{S. meliloti NodG possessed OAR activity in vitro.}

To directly test the relative activities of SmNodG and SmFabG, the proteins were expressed in E. coli BL21 (DE3) and were purified to allow direct assays of the respective OAR activities in vitro. Sodium dodecyl sulfate (SDS)-gel electrophoresis showed that $\mathrm{SmFabG}$ and SmNodG were both highly expressed (data not shown). The $\mathrm{N}$-terminal $\mathrm{His}_{6}$ tagged versions of SmFabG and SmNodG were then purified by nickel chelate chromatography, to obtain preparations that each gave a single band upon SDS-gel electrophoresis (Fig. 2A). The purified SmFabG and SmNodG proteins had monomeric molecular masses of 28 and $27 \mathrm{kDa}$, respectively, which were in good agreement with the values calculated from the sequences of the tagged proteins (FabG, 28.6 and NodG, $27.9 \mathrm{kDa})$. The E. coli fatty acid biosynthetic proteins EcFabD(malonyl-CoA:ACP transacylase), EcFabH(3-ketoacylACP synthase III), EcFabG, EcFabZ(3-hydroxyacyl-ACP dehydrase), and EcFabI(enoyl-ACP reductase), plus Vibrio harveyi acyl-ACP synthetase (AasS) and the Ralstonia solanacearum RSp0194 protein(Mao et al. 2015) were also purified by nickel chelate chromatography (data not shown). Holo-ACP protein was expressed in E. coli and purified (discussed below).
To test SmNodG function, the initiation steps of the fatty acid synthesis reaction were reconstituted by the sequential addition of EcFabD, EcFabH, OAR (EcFabG, SmNodG, or SmFabG), EcFabZ, and EcFabI, which was followed by incubation and analysis by conformationally sensitive gel electrophoresis. In the absence of OAR, only holo-ACP was seen and this was probably due to hydrolysis of the labile 3-oxobutyryl-ACP during electrophoresis (Fig. 2B, lane 5). The addition of EcFabG, SmNodG, or SmFabG to the reaction mixture resulted in the production of butyryl-ACP (Fig. 2B, lanes 2, 3, and 4). Note that OAR converts 3-oxobutyryl-ACP to 3-hydroxybutyryl-ACP, EcFabZ dehydrates 3-hydroxybutyryl-ACP to produce trans-2butenoyl-ACP, and EcFabI reduces trans-2-butenoyl-ACP to butyryl-ACP. These data clearly showed that, like E. coli FabG and SmFabG, SmNodG could complete the initial cycle of fatty acid synthesis to produce butyryl-ACP. The enzymatic activity of SmNodG during the reduction of long-chain 3-oxoacyl-ACP substrates was also tested. These reactions were reconstituted by adding malonyl-ACP, octanoyl-ACP, Ralstonia solanacearum RSp0194, OAR (EcFabG, SmNodG, or SmFabG), E. coli FabZ and FabI, and cofactors NADPH and NADH. The results showed that Ralstonia solanacearum RSp0194 could condense octanoylACP with malonyl-ACP to produce 3-oxodecanoyl-ACP. However, due to hydrolysis of the labile 3-oxodecanoyl-ACP during electrophoresis (Fig. 2C, lane 5), only octanoyl-ACP and holoACP were seen on the gel. After the addition of OAR reductase (EcFabG, SmNodG, or SmFabG) to the reaction mixture, all incubations produced the expected decanoyl-ACP (Fig. 2C, lanes 2, 3, and 4). Thus, these data further indicated that, like EcFabG and SmFabG, SmNodG could reduce long-chain 3-oxoacyl-ACP to long-chain acyl-ACP in vitro and that SmnodG encodes an OAR.

The OAR activities of SmFabG or SmNodG were also assayed using acetoacetyl-CoA as a substrate, by monitoring the decrease in NADPH absorbance at $340 \mathrm{~nm}$ (discussed below). Although

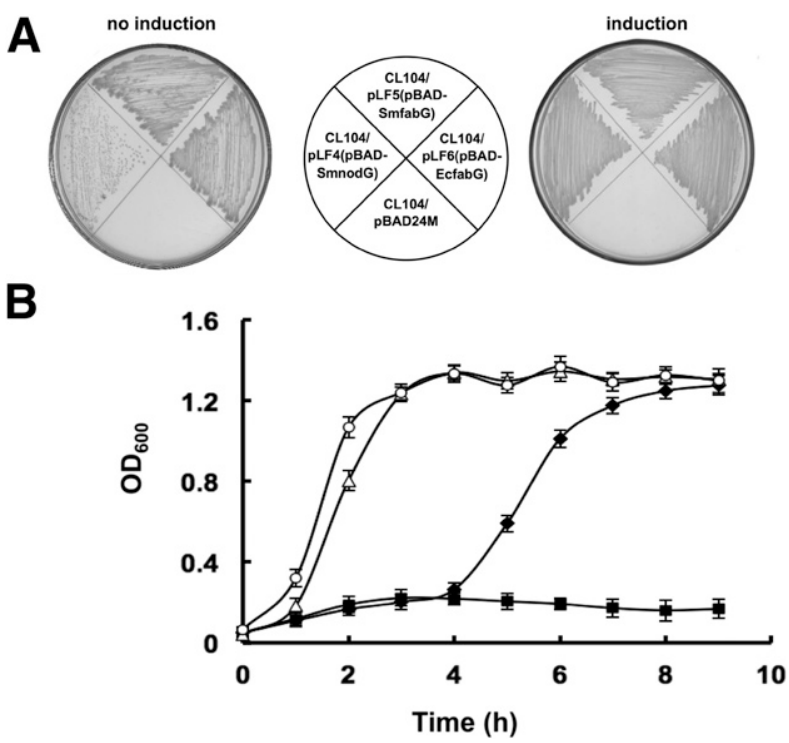

Fig. 1. Growth of Escherichia coli fabG (ts) mutant CL104 transformants containing plasmids carrying Sinorhizobium meliloti nod $G$ and fabG. A, E. coli CL104 derivatives, carrying the pBAD24M-derived plasmids pLF4 (pBAD-SmnodG), pLF5 (pBAD-SmfabG), and pLF6 (pBAD-EcfabG) were grown at $42^{\circ} \mathrm{C}$ on rich broth $(\mathrm{RB})$ plates in the absence of $0.01 \%$ arabinose (no induction) or in the presence of $0.01 \%$ arabinose (induction). B, Transformants of E. coli fabG(Ts) mutant CL104 with pBAD24M-derived plasmids grown in RB liquid containing $0.01 \%$ arabinose. $\bigcirc$, strain CL104/pLF6 (pBAD-EcfabG); $\triangle$, strain CL104/pLF5 (pBAD-SmfabG); strain CL104/pLF4 (pBAD-SmnodG); and ש, strain CL104/pBAD24M. Data are means \pm 1 standard deviation of three independent assays. 
both SmFabG and SmNodG reduced acetoacetyl-CoA, the OAR activity of $\mathrm{SmNodG}(81.5 \pm 8.5 \mu \mathrm{mol} / \mu \mathrm{g}$ per second $)$ was only $60 \%$ of SmFabG activity $(140.14 \pm 11.8 \mu \mathrm{mol} / \mu \mathrm{g}$ per second $)$. We also compared the abilities of SmFabG and SmNodG to reduce various chain length 3 -oxoacyl-ACPs, using the procedures described below (data not shown). The reduction ability of SmNodG was about $70 \%$ that of SmFabG for various chain length 3-oxoacyl-ACP. All these data explained why, in the absence of arabinose, the CL104 strain that carried pLF4 (pBADSmnodG) grew less well than the CL104 strain that carried pLF5 (SmfabG).

\section{SmnodG is not essential}

for $S$. meliloti growth and fatty acid synthesis.

We attempted to disrupt the SmnodG and $\operatorname{SmfabG}$ genes in order to determine the physiological functions of SmnodG and SmfabG during $S$. meliloti cell growth. Two pK18mobsacBborne suicide plasmids used to delete $\operatorname{Smnod} G$ or $S m f a b G$ were constructed. Plasmids carrying the in-frame gene deletion were introduced into the genome of the wild-type $S$. meliloti strain 1021 via conjugal transfer from E. coli S17-1 (Table 1). Cultures grown from the integrated colonies were then plated onto tryptone yeast (TY) medium containing sucrose, in order to select for the loss of the plasmid sequences. The successful construction of the designed mutations was assayed by polymerase chain reaction (PCR) analysis using the primers listed in Supplementary Table S1. SmnodG deletion mutant strain LF1 $(\Delta n o d G$ ) was obtained but, unfortunately, the $S m f a b G$ deletion strain was not isolated; only single-crossover integrants were obtained.
We tested the growth of LF1 ( $\Delta$ nodG) in TY medium. The data showed LF1 $(\Delta \operatorname{nod} G)$ grew as well as wild-type strain 1021 in TY medium (Fig. 3A). The fatty acid composition of strain LF1 ( $\Delta$ nod $G)$ grown in TY medium was also determined by gas chromatography-mass spectrometry (GC-MS) (Table 2). The results showed the fatty acid composition of strain LF1 $(\Delta \operatorname{nod} G)$ was similar to that of wild-type strain 1021 . These observations suggested that SmnodG was not essential for $S$. meliloti growth and that $\mathrm{SmNodG}$ may have no role in fatty acid synthesis.

\section{Expression of SmnodG}

from a plasmid replaced the function of SmfabG.

Only single-crossover integrant strains were obtained during our attempts to generate a SmfabG-deleted $S$. meliloti strain (Supplementary Fig. S2A). This result suggested that SmfabG is an essential $S$. meliloti gene. In order to examine this hypothesis and test the activity of SmNodG in vivo, we constructed plasmid pLF7 (pSRK-SmnodG) containing the wildtype SmnodG of $S$. meliloti that had been cloned into vector pSRK-Gm. This plasmid was transformed into the SmfabG single-crossover integrant strain. The conjugants were then plated on a medium containing gentamicin, sucrose, and isopropyl- $\beta$-Dthiogalactoside (IPTG), to select for loss of plasmid pK18mobsacB sequences. PCR assays showed that one of the surviving colonies, named LF4, had lost the $f a b G$ gene from its genome. The growth of strain LF4 ( $\Delta f a b G / p L F 7$ [pSRK-SmnodG]) was tested on a TY plate in the absence of IPTG. Under IPTG induction, strain LF4 ( $\triangle$ fabG/pLF7 [pSRK-SmnodG]) grew as well as wildtype strain 1021, but it failed to grow without IPTG induction
A

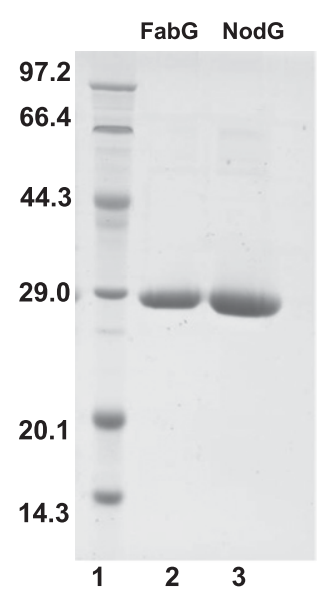

B

\begin{tabular}{|c|c|c|c|c|c|c|}
\hline- & + & + & + & + & - & EcFabH \\
\hline- & EcG & FabG & NodG & - & - & OAR \\
\hline- & + & + & + & + & - & EcFabZ \\
\hline- & + & + & + & + & - & EcFabl \\
\hline 1 & 2 & 3 & 4 & 5 & 6 & $\leftarrow$ holo-ACP \\
$-C_{4}$-ACP
\end{tabular}

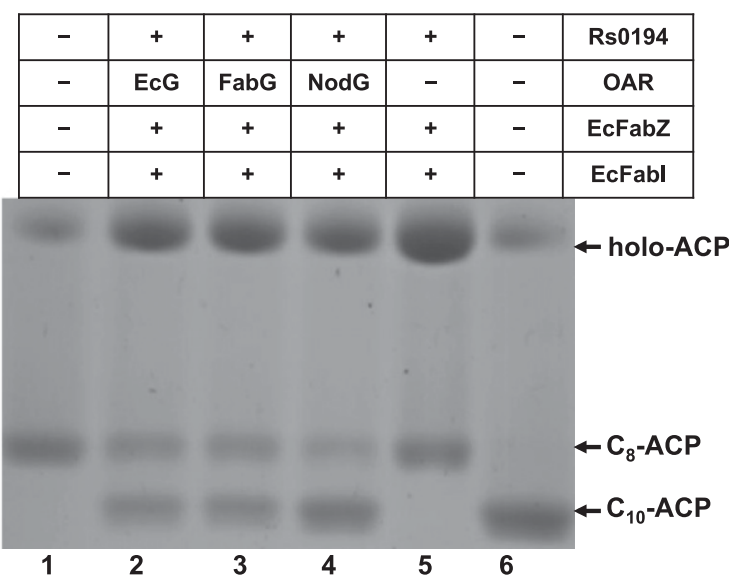

Fig. 2. Purification of Sinorhizobium meliloti FabG and NodG from Escherichia coli BL21 (DE3) and assay of the in vitro fatty acid synthesis abilities of $S$. meliloti FabG and NodG. A, Purification of $S$. meliloti FabG and NodG by native nickel-chelate chromatography. Lane 1, molecular mass markers; lane 2, $S$. meliloti-purified FabG protein; and lane 3, S. meliloti-purified NodG protein. B, Functions of $S$. meliloti FabG and NodG in the initial cycle of fatty acid synthesis. Lanes 1 and 6, holo-ACP; lane 2, reaction product containing E. coli FabG; lane 3, reaction product containing S. meliloti FabG; lane 4, reaction product containing S. meliloti NodG; and lane 5, reaction product without 3-ketoacyl-ACP reductase. C, Functions of $S$. meliloti FabG and NodG in the elongation cycle of fatty acid synthesis. Lane 1, product of octanoyl-ACP synthesized by Vibrio harveyi AasS; lane 2, reaction product catalyzed by E. coli FabG; lane 3, reaction product catalyzed by $S$. meliloti FabG; lane 4, reaction product catalyzed $S$. meliloti NodG; lane 5, reaction product without 3-ketoacyl-ACP reductase; and lane 6, product of decanoyl-ACP synthesized by $V$. harveyi AasS. EcG = E. coli FabG; OAR = 3-oxoacyl-ACP reductase; $\mathrm{EcFabH}=$ E. coli 3 -ketoacyl-ACP synthase III; EcFabZ = E. coli 3-hudroxyacyl-ACP dehydrase; and EcFabI = E. coli enoyl-ACP reductase. 
(Fig. 3B). Quantitative reverse transcription PCR analysis showed that the SmnodG expression level in strain LF4 ( $\triangle \mathrm{fabG} / \mathrm{pLF} 7$ [pSRK-SmnodG]) under IPTG induction was twofold higher than in strain LF4 ( $\Delta$ fabG/pLF7 [pSRK-SmnodG]) without IPTG induction and tenfold higher than in wild-type strain 1021 (Supplementary Fig. S5). These results indicated that $S m f a b G$ could be deleted from the genome when SmnodG from a plasmid was overexpressed.

To confirm that the failure of strain LF4 ( $\triangle f a b G / p L F 7$ [pSRK-SmnodG]) to grow in the absence of IPTG was due to cessation of fatty acid synthesis, we examined its ability to carry out fatty acid synthesis under these conditions. We prepared cell-free extracts of IPTG-induced and uninduced LF4 ( $\triangle$ fabG/pLF7 [pSRK-SmnodG]), as described below, and used them in fatty acid synthesis assays. Although uninduced cell extracts of LF4 ( $\Delta$ fabG/pLF7 [pSRK-SmnodG]) produced long-chain acyl-ACPs (Fig. 4, lane 2), the induced cell extracts were much more active (Fig. 4, lane 5). The addition of purified SmNodG (Fig. 4, lane 3) or SmFabG (Fig. 4, lane 4) to uninduced LF4 cell extracts increased acyl-ACP production. The activities of OAR in cell-free extracts were also tested using acetoacetyl-CoA as a substrate and by monitoring the decrease in NADPH absorbance at $340 \mathrm{~nm}$. The activity of OAR in the uninduced cell extracts $(23.2 \pm 2.5 \mu \mathrm{mol} / \mu \mathrm{g}$ per second) was only half the total activity observed in the induced cell extracts $(50.8 \pm 2.2 \mu \mathrm{mol} / \mu \mathrm{g}$ per second $)$. These results confirm that mutation in $S m f a b G$ decreased fatty acid production and SmNodG could substitute for SmFabG in fatty acid synthesis.

To further characterize the function of SmNodG in S. meliloti fatty acid biosynthesis in vivo, Smnod $G$ was deleted from the LF4 ( $\Delta$ fabG/pLF7 [pSRK-SmnodG]) genome to yield strain LF2 ( $\triangle$ fabG $\Delta$ nodG/pLF7 [pSRK-SmnodG]) (Supplementary Fig. S3) and a control strain, LF3 ( $\Delta$ fabG $\Delta$ nodG/pLF8 [pSRK$S m f a b G])$, that carried wild-type $S m f a b G$-encoded plasmid pLF8 (pSRK-SmfabG) (Supplementary Fig. S4) in which the genomic SmfabG and SmnodG genes had been disrupted, was also constructed. The growth rates of strains LF2 $(\Delta f a b G$

Table 1. Strains and plasmids used in this study

\begin{tabular}{|c|c|c|}
\hline Strains and plasmids & Relevant characteristics ${ }^{\mathrm{z}}$ & Source \\
\hline \multicolumn{3}{|l|}{ Escherichia coli strains } \\
\hline $\mathrm{DH} 5 \alpha$ & 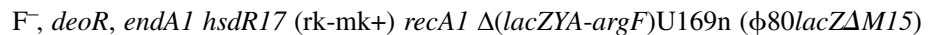 & TaKaRa \\
\hline BL21 (DE3) & $\mathrm{F}^{-}, d c m$ отр ThsdS $\left(\mathrm{rB}^{-} \mathrm{mB}^{-}\right)(\lambda \mathrm{DE} 3)$ & TaKaRa \\
\hline S17-1 & $\mathrm{F}^{-}$, thi pro hsdR (RP4-2 Tc::Mu Km::Tn7 (Tp Sm)) & CGSC \\
\hline CL104 & fabG (Ts) panD $\mathrm{Cm}^{\mathrm{r}}$, $\mathrm{Tet}^{\mathrm{r}}, \mathrm{Km}^{\mathrm{r}}$ & \\
\hline \multicolumn{3}{|c|}{ Sinorhizobium meliloti strains } \\
\hline 1021 & Wild-type strain & American Type Culture Collection \\
\hline LF1 & S. meliloti $1021 \Delta$ nodG & This study \\
\hline LF2 & S. meliloti $1021 \Delta$ nodG $\Delta f a b G$ strain carrying the SmnodG-encoding plasmid pLF7 & This study \\
\hline LF3 & S. meliloti $1021 \Delta$ nod $\Delta \triangle f a b G$ strain carrying the $S m f a b G$-encoding plasmid pLF8 & This study \\
\hline LF4 & S. meliloti $1021 \Delta f a b G$ strain carrying the SmnodG-encoding plasmid pLF7 & This study \\
\hline \multicolumn{3}{|c|}{ 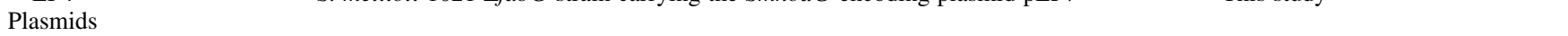 } \\
\hline pMD19 & $A m p^{r}, T$-vector & Takara \\
\hline pBAD24M & Amp ; pBAD24 NcoI site replaced by the NdeI site & Zhu et al. 2010 \\
\hline pSRK-Gm & $\begin{array}{l}\mathrm{Gm}^{\mathrm{r}} ; \mathrm{pBBR} 1 \mathrm{MCS}-2 \text {-derived broad-host-range expression vector containing the lac } \\
\text { promoter, } \text { lacI }^{q} \text {, and } \text { lacZ }^{+}\end{array}$ & Khan et al. 2008 \\
\hline pET28b & $\mathrm{Km}^{\mathrm{r}}$, expression vector & Novagen \\
\hline pK18mobsacB & $\mathrm{Km}^{\mathrm{r}} ;$ sacB-based gene replacement vector & Schäfer et al. 1994 \\
\hline pYFJ84 & Ampr, Vibrio harveyi aas $S$ cloned between the NdeI and BamHI sites of pET16b & Jiang et al. 2006 \\
\hline pFSD & $\mathrm{Km}^{\mathrm{r}}$, E.coli fabD cloned between the $N d e \mathrm{I}$ and HindIII sites of pET28b & Feng et al. 2008 \\
\hline pFSH & $\mathrm{Km}^{\mathrm{r}}$, E.coli fabH cloned between the NdeI and HindIII sites of pET28b & Feng et al. 2008 \\
\hline pFSG & $\mathrm{Km}^{\mathrm{r}}$, E.coli fabG cloned between the $N d e \mathrm{I}$ and HindIII sites of pET28b & Feng et al. 2008 \\
\hline pFSZ & $\mathrm{Km}^{\mathrm{r}}$, E.coli fabZ cloned between the NdeI and HindIII sites of pET28b & Feng et al. 2008 \\
\hline pFSI & $\mathrm{Km}^{\mathrm{r}}$, E.coli fabI cloned between the NdeI and HindIII sites of pET28b & Feng et al. 2008 \\
\hline pET-ACP & $\mathrm{Km}^{\mathrm{r}}$, E.coli acpP cloned between the $N c o \mathrm{I}$ and $B a m \mathrm{HI}$ sites of $\mathrm{pET} 28 \mathrm{~b}$ & Wang et al. 2008 \\
\hline pBAD-ACPS & $\mathrm{Cm}^{\mathrm{r}}$, E.coli acpS cloned between NdeI and HindII of pBAD34 & Wang et al. 2008 \\
\hline pYH9 & $\begin{array}{l}\mathrm{Km}^{\mathrm{r}} \text {; Ralstonia solanacearum } \mathrm{RSp} 0194 \text { cloned between the NdeI and BamHI sites } \\
\text { of pET-28b }\end{array}$ & Laboratory collection \\
\hline pLF1 & Amp ; S. meliloti 1021 nodG cloned into pMD19 & This study \\
\hline pLF2 & Amp $^{\text {r; }}$ S. meliloti 1021 fabG cloned into pMD19 & This study \\
\hline pLF3 & $\mathrm{Amp}^{\mathrm{r}} ;$ E.coli fabG cloned into pMD19 & This study \\
\hline pLF4 & $\begin{array}{l}\text { Ampr } \text {; SmnodG in pBAD24M (ligation of the NdeI-HindIII fragment from pLF1 } \\
\text { with pBAD24M at the same sites) }\end{array}$ & This study \\
\hline pLF5 & $\begin{array}{l}\text { Ampr } ; \text { SmfabG in pBAD24M (ligation of the NdeI-HindIII fragment from pLF2 } \\
\text { with pBAD24M at the same sites) }\end{array}$ & This study \\
\hline pLF6 & $\begin{array}{l}\text { Amp } p^{\mathrm{r}} ; E c f a b G \text { in pBAD24M (ligation of the NdeI-HindIII fragment from pLF3 } \\
\text { with pBAD24M at the same sites) }\end{array}$ & This study \\
\hline pLF7 & $\begin{array}{l}\mathrm{Gm}^{\mathrm{r}} \text {; SmnodG in pSRK (ligation of the NdeI-HindIII fragment from pLF1 with } \\
\text { pSRK at the same sites) }\end{array}$ & This study \\
\hline pLF8 & $\begin{array}{l}\mathrm{Gm}^{\mathrm{r}} \text {; SmfabG in pSRK(ligation of the NdeI-HindIII fragment from pLF2 with } \\
\text { pSRK at the same sites) }\end{array}$ & This study \\
\hline pLF9 & $\begin{array}{l}\mathrm{Km}^{\mathrm{r}} \text {; SmnodG from pLF1 digested with NdeI and HindIII and inserted into the } \\
\text { same sites in pET-28b }\end{array}$ & This study \\
\hline pLF10 & $\begin{array}{l}\mathrm{Km}^{\mathrm{r}} ; \text {; } m f a b G \text { from pLF2 digested with } N d e \mathrm{I} \text { and } H i n \mathrm{dIII} \text { and inserted into the same } \\
\text { sites in pET-28b }\end{array}$ & This study \\
\hline pLF11 & $\begin{array}{l}\mathrm{Km}^{\mathrm{r}} ; 1,000 \text { bp SmnodG deletion DNA fragment was cloned between } E c o \mathrm{RI} \text { and } \\
\text { HindIII sites in pK18mobscaB }\end{array}$ & This study \\
\hline pLF12 & $\begin{array}{l}\mathrm{Km}^{\mathrm{r}} ; 1,000 \text { bp SmfabG deletion DNA fragment was cloned between } E c o \mathrm{RI} \text { and } \\
\text { HindIII sites in pK18mobscaB }\end{array}$ & This study \\
\hline
\end{tabular}

\footnotetext{
${ }^{\mathrm{z}} \mathrm{Cm}^{\mathrm{r}}, \mathrm{Tet}^{\mathrm{r}}, \mathrm{Km}^{\mathrm{r}} \mathrm{Gm}^{\mathrm{r}}$, and $\mathrm{Amp}^{\mathrm{r}}$ indicate resistance to chloramphenicol, tetracycline, kanamycin, gentamicin, and ampicillin, respectively.
} 
$\Delta$ nodG/pLF7 [pSRK-SmnodG]) and LF3 ( $\triangle$ fabG $\Delta$ nodG/pLF8 [pSRK-SmfabG]) were compared with wild-type 1021 in TY medium containing IPTG. These mutant strains grew almost as well as the wild-type strain in TY medium containing IPTG (Fig. 3A). Analysis of the fatty acid composition of strain LF2 ( $\Delta$ fabG $\Delta$ nodG/pLF7 [pSRK-SmnodG]) and LF3 $(\Delta f a b G$ $\Delta$ nod $G / \mathrm{pLF} 8$ [pSRK-SmfabG]) showed that both strains contained almost the same species and amounts of fatty acid as the wild-type strain 1021 (Table 2). These results confirmed that, like SmfabG, SmnodG encodes OAR and overexpression of

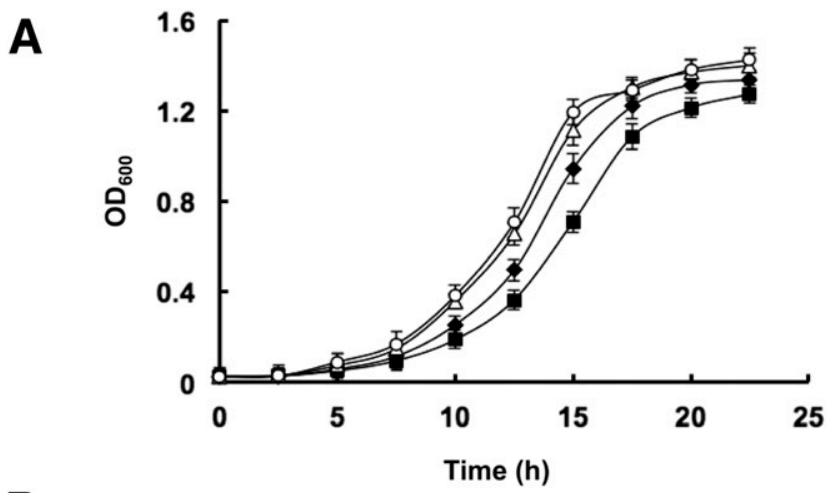

B

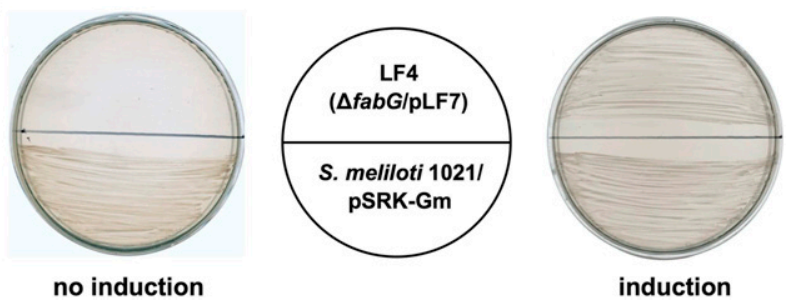

Fig. 3. Growth of Sinorhizobium meliloti fabG or nodG deletion strains in tryptone yeast (TY) medium. A, Growth curves of $S$. meliloti mutant strains LF1 ( $\operatorname{nnodG),~LF2~(~} \Delta f a b G \Delta$ nodG/pLF7[pSRK-SmnodG]), and LF3 $(\triangle f a b G \Delta$ nodG/pLF8 [pSRK-SmfabG]) cultured in TY liquid medium. Isopropyl- $\beta$-D-thiogalactoside (IPTG) was added to the TY medium for strains LF2 ( $\triangle f a b G \quad \Delta$ nodG/pLF7 [pSRK-SmnodG]) and LF3 $(\Delta f a b G$ $\Delta$ nodG/pLF8 [pSRK-SmfabG]). O, wild-type strain 1021-carried vector pSRK-Gm; $\triangle$, S. meliloti nodG deletion strain LF1 $(\Delta$ nodG); $\bullet$, S. meliloti mutant LF3 $(\Delta f a b G \Delta$ nodG/pLF8 [pSRK-SmfabG]); and $\mathbf{m}$, mutant strain LF2 ( $\triangle$ fabG $\Delta$ nodG/pLF7 [pSRK-SmnodG]). Data are means \pm 1 standard deviation of three independent assays. B, Growth of mutant strain LF4 $(\Delta f a b G / p L F 7$ [pSRK-SmnodG]) on TY medium plates containing no IPTG (no induction) or IPTG (induction). S. meliloti 1021/pSRK-Gm indicates wild-type strain $S$. meliloti 1021 carried vector pSRK-Gm.
SmnodG produced enough OAR to allow the $S$. meliloti fabG mutant to synthesize fatty acids.

\section{Overexpression of SmnodG affected growth when \\ $S$. meliloti was subjected to various environmental stresses.}

Within the soil and during nodulation, S. meliloti copes with various stresses imposed by the environment or by plant responses to bacterial invasion, including salinity, $\mathrm{pH}$, reactive oxygen, and more (Fernandez-Aunión et al. 2010; Fontenelle et al. 2011; Miller-Williams et al. 2006; Patankar and González 2009). It has been reported that mutation in $f a b G$ rendered $S$. meliloti unable to tolerate high salt (Miller-Williams et al. 2006). To further investigate SmNodG effects, we tested the growth of the mutant strains on TY plates under various environmental stresses (high $\mathrm{NaCl}$ concentrations, high SDS concentrations, high $\mathrm{H}_{2} \mathrm{O}_{2}$ concentrations, and low $\mathrm{pH}$ values). The results showed that the growth rates of strain LF1 $(\Delta \operatorname{nod} G)$ and LF3 ( $\Delta$ fabG $\Delta$ nodG/pLF8 [pSRK-SmfabG]) were similar to that of the wild-type strain 1021 under these stress conditions (Fig. 5). Mutant strain LF2 ( $\triangle$ fabG $\Delta$ nodG/pLF7 [pSRKSmnodG]) was more resistant to high concentrations of $\mathrm{NaCl}$ (Fig. 5A) and $\mathrm{H}_{2} \mathrm{O}_{2}$ (Fig. 5C) and more sensitive to high concentrations of SDS (Fig. 5B) than the wild-type strain 1021.

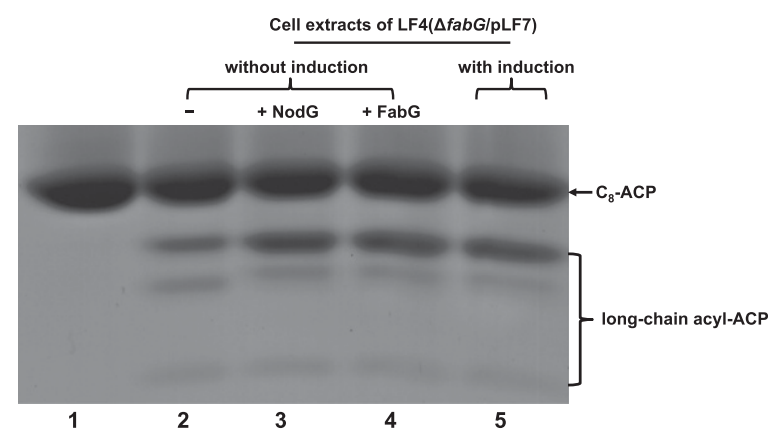

Fig. 4. Analysis of fatty acid synthesis in cell-free extracts of LF4 $(\triangle f a b G / p L F 7$ [pSRK-SmnodG]). Lane 1, product of octanoyl-ACP synthesized by Vibrio harveyi AasS; lane 2, reaction product catalyzed by cell-free extracts of LF4 ( $\triangle f a b G / \mathrm{pLF} 7$ [pSRK-Smnod $G$ ]) under no isopropyl- $\beta$-Dthiogalactoside (IPTG) induction; lane 3, reaction product catalyzed by cellfree extracts of LF4 ( $\triangle f a b G / \mathrm{pLF} 7$ [pSRK-Smnod $G$ ] ) grown without IPTG induction but containing purified SmNodG protein; lane 4, the reaction product catalyzed by cell-free extracts of LF4 $(\Delta f a b G / p L F 7$ [pSRKSmnodG]) grown without IPTG induction but containing purified SmFabG protein; and lane 5, reaction product catalyzed by cell-free extracts of LF4 $(\Delta f a b G / p L F 7$ [pSRK-Smnod $G])$ grown with IPTG induction.

Table 2. Fatty acid compositions of the total lipid extracts from Sinorhizobium meliloti nodG mutants ${ }^{\mathrm{y}}$

\begin{tabular}{|c|c|c|c|c|}
\hline \multirow[b]{2}{*}{ Fatty $\operatorname{acids}^{z}$} & \multicolumn{4}{|c|}{ Composition (\%) } \\
\hline & LF1 $(\Delta \operatorname{nod} G)$ & LF2 ( $\Delta$ fabG $\Delta$ nodG/pLF7) & LF3 ( $\Delta$ fabG $\Delta$ nodG/pLF8) & Wild type \\
\hline$n-3-O H-C_{14: 0}$ & $15.86 \pm 1.50$ & $14.45 \pm 1.09$ & $15.15 \pm 0.96$ & $15.17 \pm 2.29$ \\
\hline$n-C_{16: 0}$ & $6.65 \pm 1.50$ & $7.43 \pm 0.70$ & $7.22 \pm 0.05$ & $7.14 \pm 0.37$ \\
\hline$n-C_{16: 1}$ & $1.00 \pm 0.04 \mathrm{a}$ & $0.79 \pm 0.06 b$ & $0.86 \pm 0.03 b$ & $0.96 \pm 0.08 \mathrm{a}$ \\
\hline $\mathrm{n}-3-\mathrm{OH}-\mathrm{C}_{16: 0}$ & $0.79 \pm 0.07$ & $0.73 \pm 0.02$ & $0.76 \pm 0.03$ & $0.70 \pm 0.11$ \\
\hline $\mathrm{n}-\mathrm{C}_{18.0}$ & $2.29 \pm 0.17$ & $2.42 \pm 0.46$ & $2.32 \pm 0.16$ & $2.19 \pm 0.56$ \\
\hline$n-C_{18: 1}$ & $60.47 \pm 1.11$ & $60.11 \pm 1.01$ & $61.56 \pm 1.14$ & $61.51 \pm 1.48$ \\
\hline $\mathrm{n}-3-\mathrm{OH}-\mathrm{C}_{18: 0}$ & $5.26 \pm 0.21$ & $5.24 \pm 0.27$ & $5.06 \pm 0.21$ & $5.09 \pm 0.33$ \\
\hline $\mathrm{n}-\mathrm{C}_{18: 2}$ & $3.54 \pm 0.44$ & $3.14 \pm 0.43$ & $2.88 \pm 0.10$ & $2.83 \pm 0.17$ \\
\hline$n-C_{19: 0}$ cyclo & $4.13 \pm 0.25 b$ & $5.69 \pm 0.59 \mathrm{a}$ & $4.20 \pm 0.22 b$ & $4.41 \pm 0.60 \mathrm{~b}$ \\
\hline
\end{tabular}

${ }^{\mathrm{y}}$ Cells were grown on tryptone yeast medium for $48 \mathrm{~h}$ at $28^{\circ} \mathrm{C}$. The total lipids were extracted and transesterified to obtain fatty acid methyl esters, and the products were identified by gas chromatography-mass spectrometry. The values are percentages of total fatty acids and are the means \pm the standard deviations of three independent experiments. Different letters indicate significant differences $(P<0.05)$ according to Fisher's least significant difference test.

${ }^{\mathrm{z}} \mathrm{n}$-3-OH- $\mathrm{C}_{14: 0}=3$-hydroxytetradecanoic. $\mathrm{n}-\mathrm{C}_{16: 0}=$ hexadecanoic, $\mathrm{n}-\mathrm{C}_{16: 1}=$ cis-9-hexadecenoic, $\mathrm{n}$-3-OH-C $\mathrm{C}_{16: 0}=3$-hydroxyhexadecanoic, $\mathrm{n}-\mathrm{C}_{18: 0}=$ octadecanoic, $\mathrm{n}-\mathrm{C}_{18: 1}=$ cis-11-octadecenoic, $\mathrm{n}-3-\mathrm{OH}-\mathrm{C}_{18: 0}=3$-hydroxyoctadecanoic, $\mathrm{n}-\mathrm{C}_{18: 2}=$ cis-11-cis-9-octadecenoic, and $\mathrm{n}-\mathrm{C}_{19: 0}$ cyclo $=$ cis-11,12 methyleneoctadecanoic acids. 
These data suggested that overexpression of SmnodG helps $S$. meliloti to adapt to environmental changes.

\section{Deletion of SmnodG caused delayed alfalfa nodulation.}

Previous research suggested that $S$. meliloti nod $G$ mutants inoculated on alfalfa either did not negatively affect nodulation (Swanson et al. 1987) or caused only a slight delay in nodulation (Horvath et al. 1986). To confirm this point, symbiosis nodulation was tested under nitrogen-deficient conditions on alfalfa seedlings in both liquid medium and vermiculite. In the liquid test, the nodulation rate was assayed by counting the numbers of nodules at particular time points after inoculation (Fig. 6A). These data showed that, although the first nodules were observed on the wild-type 1021 and the LF1 (nodG) at about the same time (7 days after inoculation), the mutant LF1 (nodG) showed a slight reduction in nodule number compared with the wild type. This was consistent with previous research and confirmed that Smnod $G$ was not essential to alfalfa nodulation. Compared with wild-type 1021 , both double-deletion strains, LF2 ( $\triangle \mathrm{fabG}$
$\Delta$ nodG/pLF7 [pSRK-SmnodG]) and LF3 ( $\Delta$ fabG $\Delta$ nodG/pLF8 [pSRK-SmfabG]), drastically delayed nodulation, and nodule formation started only about 14 days after inoculation. This was probably due to the low activities of OAR in S. meliloti mutants, since the expression of OAR in both deletion strains was under the control of the E. coli lac promoter but, during alfalfa nodulation, there was no IPTG to induce its expression. It should be noted that the nodulation rate of LF3 $(\Delta f a b G \Delta$ nodG/pLF8 [pSRK-SmfabG]) was much lower than that of LF2 ( $\triangle \mathrm{fabG}$ $\Delta$ nodG/pLF7 [pSRK-SmnodG]). This implied that SmnodG was able to increase alfalfa nodulation efficiency. The above results were also confirmed by the vermiculite test. In the vermiculite test, the average number of nodules per plant and the percentage of pink nodules were determined using 6-week-old alfalfa plants for each of the four bacterial strains. Although the average number of nodules per plant formed by LF1 ( $\Delta$ nodG) was less than that formed by the wild-type 1021, the percentage of pink nodules was almost the same in these two strains. However, for the double-deletion strains, LF2 ( $\Delta$ fabG $\Delta$ nodG/pLF7 [pSRK-SmnodG])
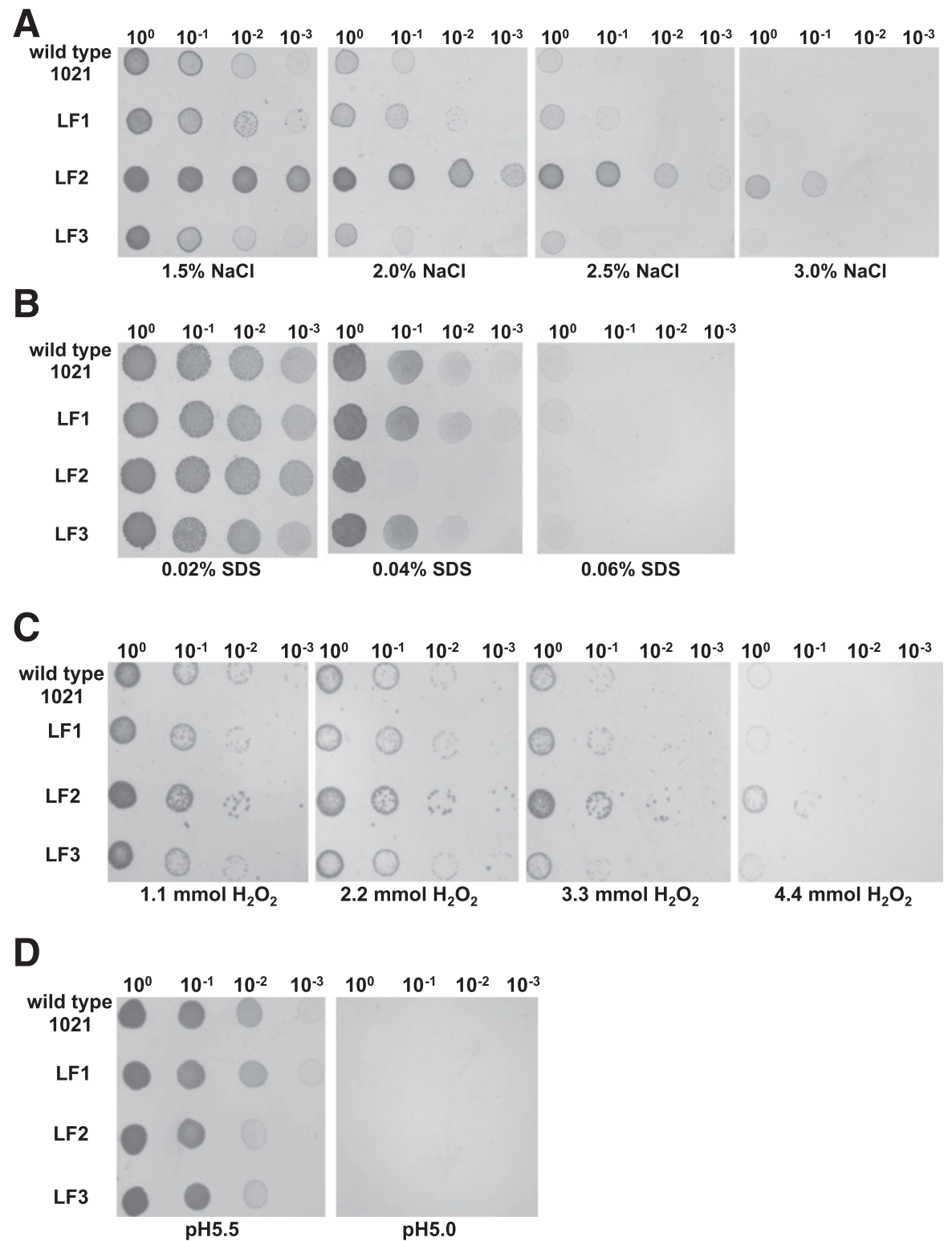

Fig. 5. Growth of Sinorhizobium meliloti mutant strains in adverse environments. S. meliloti mutant strains grew on tryptone yeast (TY) plates containing increasing levels of $\mathbf{A}, \mathrm{NaCl} ; \mathbf{B}$, sodium dodecyl sulfate (SDS); $\mathbf{C}, \mathrm{H}_{2} \mathrm{O}_{2}$; and $\mathbf{D}$, acidity. Cultures of $S$. meliloti mutant strains that had reached the exponential growth phase in TY liquid at $28^{\circ} \mathrm{C}$ were serially diluted, and then, $5 \mu \mathrm{l}$ of the appropriate culture dilutions was added to the TY plates containing various concentrations of $\mathrm{NaCl}$, SDS, or $\mathrm{H}_{2} \mathrm{O}_{2}$. The TY plates were adjusted to different $\mathrm{pH}$ values with $1 \mathrm{M} \mathrm{HCl}$. All plates were incubated at $28^{\circ} \mathrm{C}$ for 3 days. 
and LF3 ( $\triangle$ fabG $\Delta$ nodG/pLF8 [pSRK-SmfabG]), the average number of nodules per plant and the percentage of pink nodules were much less than the wild-type strain 1021. Moreover, a comparison of LF2 ( $\triangle$ fabG $\Delta$ nodG/pLF7 [pSRK-SmnodG]) with LF3 ( $\Delta f a b G \Delta$ nodG/pLF8 [pSRK-SmfabG]) showed that the LF2 strain had much higher average number of nodules per plant and percentage of pink nodules than LF3.

\section{DISCUSSION}

In the bacterial fatty acid synthetic pathways studied to date, only a single enzyme, FabG, had been found to catalyze the reduction of 3-oxoacyl-ACPs to 3-hydroxy acyl-ACPs (Campbell and Cronan 2001; White et al. 2005). However, it has been proposed for some time that rhizobia NodG proteins function as OARs. Although López-Lara and Geiger (2001) observed that both Rhizobium leguminosarum $\mathrm{FabG}$ and NodG had almost the same OAR activity in vitro, when using acetoacetyl-CoA as a substrate, and suggested that NodG functions as OAR, to date there has been no further evidence to confirm that Rhizobium leguminosarum NodG plays a role in fatty acid synthesis in vivo. This study found that $S$. meliloti nodG improved the growth of $E$. coli fabG(Ts) strain CL104 (Lai and Cronan 2004) and catalyzed all of the oxoacyl reduction steps in the elongation cycle of FAS II in vitro. Moreover, when $\operatorname{nod} G$ from a plasmid was overexpressed, both the $f a b G$ and nod $G$ genes could be deleted from the $S$. meliloti genome and the mutant strain still produced similar species and amounts of fatty acids to wild-type strain 1021. These results demonstrated that $S$. meliloti nodG encodes OAR and is able to functionally replace S. meliloti FabG in fatty acid synthesis and that $S$. meliloti possesses two OARs.

SmNodG and SmFabG seem to be distinct proteins, even though $S$. meliloti NodG is $79.6 \%$ identical to $S$ meliloti $\mathrm{FabG}$, and the SDR family catalytically active triad (Ser, Tyr, and Lys) and the N-terminal cofactor binding sequence (Gly motif [GXXXGXG]) are conserved in NodG protein. It seems that $f a b G$ is required for growth and fatty acid synthesis in $S$. meliloti and cannot be deleted from the chromosome, whereas nodG is not essential and the strain in which nod $G$ had been disrupted remained viable and produced similar species and amounts of fatty acids to the wild-type strain. Second, although $S m n o d G$ was not essential for alfalfa nodulation, SmnodG mutation did cause delays in alfalfa nodulation. Third, overexpression of SmnodG from a plasmid in which there had been a double deletion of the $\operatorname{fab} G$ and $\operatorname{nod} G$ background could enhance $S$. meliloti resistance to high concentrations of $\mathrm{NaCl}$ and $\mathrm{H}_{2} \mathrm{O}_{2}$.

The difference between SmFabG and SmNodG seems to be the level of OAR activity. Since the enzyme activity of SmNodG was only $70 \%$ of SmFabG in vitro and nodG was not transcribed under free-living conditions (Maillet et al. 1990), $f a b G$ could only be deleted from the chromosome in the nodD3 mutant, which was similar to the $f a b G:$ Tn 5 mutant obtained by Miller-Williams et al. (2006). However, when $\operatorname{nod} G$ from a plasmid was overexpressed, the chromosomal fabG gene was readily disrupted, because the elevated nodG expression increased OAR activity (Fig. 3B).

Although nod $G$ was not required for $\alpha, \beta$-unsaturated fatty acid synthesis (Demont et al. 1993), OAR still participates in the reduction of 3-oxo-fatty acid intermediates in $\alpha$, $\beta$-unsaturated fatty acid synthesis (Demont-Caulet et al. 1999). Furthermore, nod $G$ expression was also induced by alfalfa roots that secrete specific flavonoids (Maillet et al. 1990). These results indicated that nodulation on alfalfa roots by $S$. meliloti required high levels of OAR activity. We examined the activity of OAR in $S$. meliloti cells cultured under flavonoid induction and found that the activity of OAR in cells under flavonoid induction $(61.6 \pm 8.5 \mu \mathrm{mol} / \mu \mathrm{g}$ per second $)$ was higher than in cells without flavonoid induction $(45.3 \pm 5.6 \mu \mathrm{mol} / \mu \mathrm{g}$ per second) (data not shown). Thus, the slight delay in the nodulation of alfalfa roots caused by the nod $G$ mutant was mainly due to the low activity of OAR in the nod $G$ mutant. Other evidence supporting this point came from the observation that $S$. meliloti fabG::Tn5 lost its ability to compete for nodule occupancy (Miller-Williams et al. 2006) when NodG was the only OAR. Based on these observations, we speculate that it is the OAR activity level rather than the fabG and nodG genes or their products that is important in S. meliloti.

NodG displayed some substrate specificity during $\alpha$, $\beta$-unsaturated fatty acid synthesis. $S$. meliloti strains that lack Smnod $G$, such as LF1 ( $\Delta \operatorname{nod} G)$ and $(\Delta f a b G \Delta \operatorname{nod} G / \mathrm{pLF} 8)$, showed lower nodulation rates and percentages of pink nodules than wild-type strain 1021(Fig. 6).

\section{MATERIALS AND METHODS}

The supply sources were as follows. Malonyl-CoA, acetylCoA, fatty acids, NADH, NADPH, and the antibiotics were obtained from Sigma-Aldrich; Takara Biotechnology (Dalian) Co., Ltd. provided the molecular biology reagents; Invitrogen provided the $\mathrm{Ni}^{2+}$-agarose column; and Bio-Rad provided the Quick Start Bradford dye reagent. All other reagents were of the highest available quality.

\section{Bacterial strains, plasmids, and growth conditions.}

The strains and plasmid vectors used in this study are listed in Table 1. Luria-Bertani (LB) medium was used as the medium for $E$. coli. The E. coli fab strain phenotypes were assessed on RB medium (Ulrich et al. 1983). S. meliloti were grown on TY medium or TY agar (TY broth plus $1.5 \%$ agar) at $28^{\circ} \mathrm{C}$. Antibiotics were used at the following concentrations (in $\mu \mathrm{g} / \mathrm{ml}$ ): sodium ampicillin, 100; chloramphenicol, 30; kanamycin sulfate, 30; gentamycin, 10 (for E. coli) or 30 (for S. meliloti). IPTG was used at a final concentration of $1 \mathrm{mM}$ and L-arabinose was used at a final concentration of $0.01 \%$.

\section{Plasmid construction.}

$S m f a b G$ and SmnodG were amplified from genomic DNA obtained from wild-type strain $S$. meliloti 1021 . The primers are listed in Table 2. The PCR was performed with Pfu DNA polymerase and the products were inserted into T-vector pMD19, to give plasmid pLF1 (SmnodG) and pLF2 (SmfabG), respectively. The pLF3 that contained the E.coli fabG, which had been inserted into T-vector pMD19, was also obtained using a similar approach. All the genes in the T-vectors were confirmed by sequencing performed by Shanghai Sangon, Inc. The SmnodG in pLF1 and SmfabG in pLF2 were digested with $N d e$ I and HindIII, and the appropriate fragments were isolated and ligated into pBAD24M (Zhu et al. 2010) to create plasmids pLF4 (pBAD-SmnodG) and pLF5 (pBAD-SmfabG). They were also inserted into pSRK-Gm (Khan et al. 2008) to give pLF7 (pSRK-SmnodG) and pLF8 (pSRK-SmfabG) and into pET28b to give pLF9 (pET-SmnodG) and pLF10 (pET-SmfabG). Using the same process, we also constructed plasmid pLF6 (pBAD$E c f a b G)$, which contained E. coli fabG from pLF3.

\section{Disruption of the $f a b G$ and nodG genes.}

To in-frame delete the genomic $S$. meliloti nodG gene, a suicide plasmid was constructed as follows. The 500-bp DNA fragments upstream and downstream of nodG (called nodGUp and nodGDn) were amplified with $P f u$ DNA polymerase, using S. meliloti genomic DNA as the template and either RmnodG up1 EcoRI and RmnodG up2 (for nodGUp) or RmnodG down1 
and RmnodG down2 BamHI (for nodGDn) as the primers (Table 2), respectively. These PCR products were purified, and an overlap extension was carried out using RmnodG up1 EcoRI and RmnodG down2 BamHI as the primers. The plasmid pK18mobsacB (Schäfer et al. 1994) was digested by EcoRI and BamHI and was ligated with the 1,000-bp DNA knockout cassette fragment that had been digested using the same enzymes to yield plasmid pLF11. After mating a derivative of E. coli S17-1 carrying the pLF11 plasmid with S. meliloti 1021 on TY plates for $48 \mathrm{~h}$ at $28^{\circ} \mathrm{C}$ Supplementary Fig. S1A, the cells were suspended in TY medium and, after appropriate dilution, were spread on TY plates containing chloramphenicol plus kanamycin to select for the single-crossover integrants, which carried kanamycin resistance from the plasmid pLF11. Several single-crossover integrant colonies were inoculated into TY medium without kanamycin. All the cultures were incubated at $28^{\circ} \mathrm{C}$ for $48 \mathrm{~h}$, and then, appropriate dilutions of the cultures were spread onto TY plates containing a final concentration of $10 \%$ sucrose, to select for the deletion mutants. Colonies sensitive to kanamycin were screened by PCR, utilizing the primers listed in Table 2, and the nod $G$ deletion strain LF1 ( $\Delta$ nodG) was obtained.

To construct an in-frame deletion of SmfabG from the $S$. meliloti genome, suicide plasmid pLF12 was also constructed by the same method used to make pLF11. Unfortunately, after mating $S$. meliloti 1021 with $E$. coli S17-1, which carried pLF12, we were unable to isolate the $\operatorname{SmfabG}$ deletion strain; only singlecrossover integrants were obtained. To obtain the SmfabG deletion strain, plasmid pLF7 (pSRK-SmnodG), in which the expression of $S$. meliloti SmnodG was under the control of the E. coli lac promoter, was introduced into the SmfabG single-crossover integrant strain by conjugation. The conjugants were then plated on a medium containing gentamicin, $10 \%$ sucrose, and IPTG to select for loss of plasmid pK18mobsacB sequences. Colonies resistant to gentamicin were screened by PCR assays, using the primer pair listed in Table 2, and the results showed that the screening had isolated strain LF4 ( $\triangle f a b G / p L F 7$ [pSRK-Smnod $G])$. In this strain, the $\operatorname{SmfabG}$ had been in-frame deleted from the genome. Following mating of a derivative of E. coli S17-1 carrying pLF11 plasmid with strain LF4 ( $\Delta$ fabG/pLF7 [pSRK-SmnodG]), we successfully deleted nod $G$ from strain LF4 $(\Delta f a b G / \mathrm{pLF} 7$ [pSRKSmnodG]) and got strain LF2 ( $\triangle f a b G \Delta$ nodG/pLF7 [pSRKSmnod $G]$ ). Moreover, using a similar approach, we were able to construct LF3 ( $\Delta f a b G \Delta$ nodG/pLF8 [pSRK-SmfabG]). All deletion genes were confirmed by sequencing the PCR fragment of the disruption allele.

\section{Expression and purification of proteins.}

The pET28b-derived plasmids, pLF9 (pET-SmnodG) and pLF10 (pET-SmfabG), were introduced into E. coli BL21 (DE3), and the respective proteins, $\mathrm{SmFabG}$ and $\mathrm{SmNodG}$, were highly expressed (data not shown). The SmFabG and SmNodG were purified by nickel chelate chromatography as described previously (Zhu et al. 2010). The E. coli FabD, FabH, FabG, FabZ, and FabI V. harveyi AasS, Ralstonia solanacearum RSp0194 protein (Mao et al. 2015) and E. coli holo-ACP were also purified as described previously (Zhu et al. 2010).

\section{Assay of SmFabG and NodG activities in vitro.}

First, malonyl-ACP was synthesized from holo-ACP and malonyl-CoA using E. coli FabD, and octanoyl-ACP or decanoylACP was synthesized from octanoic acid or decanoic acid, ATP, and holo-ACP using $V$. harveyi acyl-ACP synthetase, as described previously (Jiang et al. 2006). The ability to reduce 3-oxobutyryl-ACP to 3-hydroxybutyryl-ACP by SmNodG or SnFabG during the initiation reaction of the fatty acid synthesis cycle was assessed in reaction mixtures containing
$0.1 \mathrm{M}$ sodium phosphate ( $\mathrm{pH} 7.0) ; 0.1 \mu \mathrm{g}$ each of $E$. coli $\mathrm{FabH}$, FabZ, and FabI; $50 \mu \mathrm{M}$ NADH; $50 \mu \mathrm{M}$ NADPH; $1 \mathrm{mM}$ $\beta$-mercaptoethanol; $100 \mu \mathrm{M}$ acetyl-CoA; $100 \mu \mathrm{M}$ malonyl$\mathrm{CoA}$; and $50 \mu \mathrm{M}$ holo-ACP in a final volume of $40 \mu \mathrm{l}$. The reaction mixtures used to investigate the reduction of longchain 3-ketoacyl-ACP contained $0.1 \mathrm{M}$ sodium phosphate $(\mathrm{pH}$ 7.0); $50 \mu \mathrm{M}$ malonyl-ACP; $50 \mu \mathrm{M}$ long-chain octanoyl-ACP; $0.1 \mu \mathrm{g}$ each of Ralstonia solanacearum RSp0194 protein, E. coli FabZ, and FabI; $50 \mu \mathrm{M}$ NADH; and $50 \mu \mathrm{M}$ NADPH. The reactions were initiated by the addition of $0.1 \mu \mathrm{g} \mathrm{SmFabG}$ or $\mathrm{SmNodG}$, followed by incubation for $1 \mathrm{~h}$. The reaction products were resolved by conformationally sensitive gel electrophoresis on $20 \%$ polyacrylamide gel containing a concentration of urea optimized for the separation. The gel was stained with Coomassie brilliant blue R250.

We also tested fatty acid synthesis by the cell-free extracts from strain LF4 ( $\triangle f a b G / \mathrm{pLF} 7)$. The reaction mixture contained cell-free extracts, holo-ACP, malonyl-CoA, octanoyl-ACP, NADH, and NADPH in sodium phosphate buffer $(\mathrm{pH} 7.0)$.

\section{NADPH oxidation assay.}

UV-vis spectrophotometry was used to measure OAR activity via the decrease in absorbance at $340 \mathrm{~nm}$, using an NADPH extinction coefficient of $6,220 \mathrm{M}^{-1}$. Each reaction was performed in UV-transparent microcuvettes. The reaction mixture contained $0.5 \mathrm{mM}$ acetoacetyl-CoA, $0.2 \mathrm{mM}$ NADPH, 0.1 M sodium phosphate buffer ( $\mathrm{pH} 7.4$ ), and $10 \mu \mathrm{g}$ of a purified His-tagged SmFabG, SmNodG protein, or a cellfree extract of the mutant strain. When testing the enzymatic
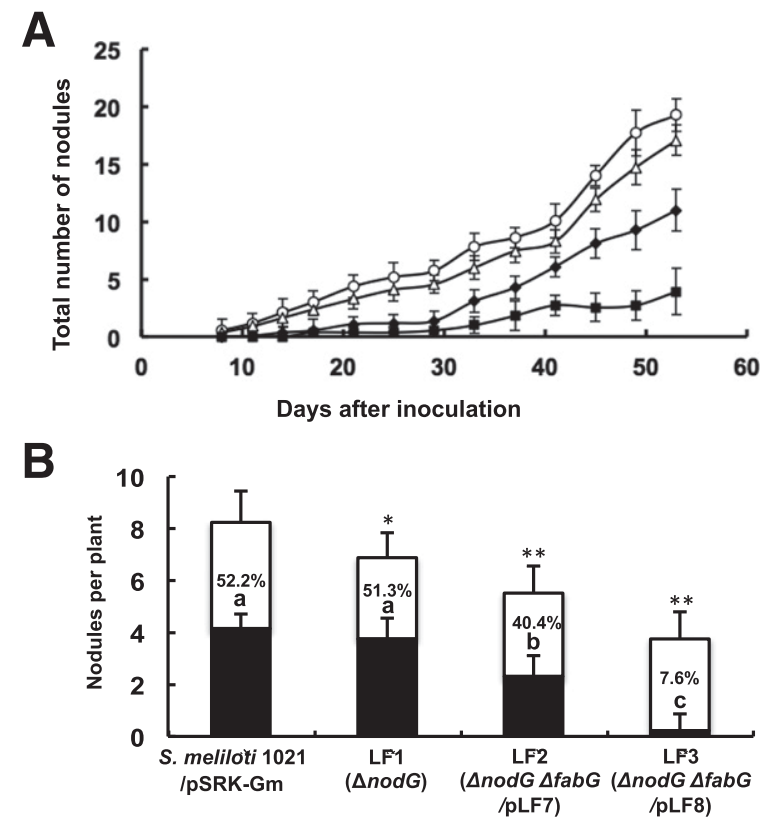

Fig. 6. Nodulation efficiency of Sinorhizobium meliloti mutant strains. A, The ability of wild type and mutant strains of $S$. meliloti to develop nodules was examined daily after the bacteria had been inoculated onto the seedlings. $\bigcirc$ indicates wild-type strain 1021-carried vector pSRK-Gm; $\triangle$, $S$. meliloti nod $G$ deletion strain LF1 $(\Delta \operatorname{nod} G) ; \boldsymbol{\Delta}, S$. meliloti mutant LF2 $(\Delta$ fabG $\Delta$ nodG/pLF7 [pSRK-SmnodG]); and $\mathbf{a}$, mutant strain LF3 $(\Delta f a b G$ $\Delta$ nodG/pLF8 [pSRK-SmfabG]). Data are means \pm 1 standard deviation of three independent experiments. B, The average number of nodules per plant and the percentage of pink nodules were determined for each of the four bacteria strains, using the vermiculite test. Bars represent standard deviations from two independent experiments with eight replicates each. Pairwise comparisons of the average of number of nodules per plant were made between the wild-type 1021 and each mutant strain by Student's $t$ test. one asterisk (*) indicates significant difference, $P<0.05$ and two $(* *)$ indicates highly significant difference, $P<0.01$. Different letters indicate significant differences $(P<0.05)$ found by Fisher's least significant difference test. 
abilities of SmFabG and SmNodG to reduce various 3-oxoacyl$\mathrm{ACP}$ chains, the reaction mixture contained acyl-CoAs $\left(\mathrm{C}_{2}-\mathrm{CoA}\right.$ to $\mathrm{C}_{10}-\mathrm{CoA}$ ) and malonyl-CoA as substrates. E. coli $\mathrm{FabD}$ and Ralstonia solanacearum RSp0194 were also added to the reaction mixture.

\section{Cell-free extract preparation.}

Cell-free extracts of $S$. meliloti strains were prepared from exponentially growing cells (optical density at $600 \mathrm{~nm}$ $\left[\mathrm{OD}_{600}\right]$ of 0.8 to 1.0$)$. The cell cultures were harvested by centrifugation and were then suspended in lysis buffer $(0.1 \mathrm{M}$ sodium phosphate, $\mathrm{pH} 7.5,5 \mathrm{mM} \beta$-mercaptoethanol, $1 \mathrm{mM}$ EDTA). The cell lysates were prepared by passing the cell suspensions three times through a French pressure cell. Cell debris was removed by ultracentrifugation for $1 \mathrm{~h}$ at $260,000 \times$ $g$, and the supernatants were dialyzed against lysis buffer for $24 \mathrm{~h}$ and were saved as cell extracts. Two kinds of cell-free extracts of strain LF4 ( $\Delta$ fabG/pLF7 [pSRK-SmnodG]) were prepared. First, we inoculated LF4 in TY liquid under IPTG induction. When the $\mathrm{OD}_{600}$ was about 0.6 , the cells were harvested and were divided into two groups. One group was transferred into fresh TY supplemented with $1 \mathrm{mM}$ IPTG; the other group was washed three times with TY to remove residual IPTG and was then transferred into fresh TY without IPTG. Both cells were allowed to grow for several hours at $28^{\circ} \mathrm{C}$ before harvest.

\section{Analysis of phospholipid compositions.}

The cultures were grown aerobically at $28^{\circ} \mathrm{C}$ in TY medium for $48 \mathrm{~h}$. Cells were then harvested from $10 \mathrm{ml}$ of cultures and were washed with fresh TY medium at room temperature. Fatty acid methyl esters were extracted according to a previously reported procedure (Zhu et al. 2010), and fatty acid methyl esters of each strain were analyzed by GC-MS as previously described (Basconcillo and McCarry 2008).

\section{Plant nodulation assays.}

Plant nodulation assays were carried out as described previously (Fontenelle et al. 2011), with some modifications. Medicago sativa (alfalfa) was used as a host plant to test nodulation by the $S$. meliloti strains. Agar slant was replaced with filter paper (Whatman Grade 1, $125 \times 125 \mathrm{~mm}$ ) to support plants grown in the nitrogen-free medium. Surface-sterilized germinating seedlings were grown on filter paper that was half soaked in $20 \mathrm{ml}$ of nitrogen-free medium in test tubes $(30 \times$ $200 \mathrm{~mm}$ ). Each tube contained one seedling. Ten 1-week-old plants were inoculated with $10^{9}$ cells of either wild type or mutant $S$. meliloti. The seedlings were kept in a growth chamber $\left(16 \mathrm{~h}\right.$ of light and $8 \mathrm{~h}$ of dark at $\left.25^{\circ} \mathrm{C}\right)$. After inoculation, the number of nodulated plants was recorded daily for one and a half months. We also performed the vermiculite test to record average number of nodules per plant and the percentage of pink nodules as described previously (Jacob et al. 2008). Briefly, six seedlings were planted in each pot and were kept in the greenhouse. When the seedlings were 1 week old, each pot was inoculated with $5 \mathrm{ml}$ of a 1:50 water dilution of TY culture saturated with either the $S$. meliloti wild type or mutant strains. Eight pot plants were infected by each strain. The plants were grown for 6 weeks, and then, their nodulation phenotypes were scored.

\section{Statistical analysis.}

The data were subjected to a one-way analysis of variance, followed by a comparison of multiple treatment levels with the control by using Fisher's least significant difference test. All statistical analyses were performed by using Microsoft Excel.

\section{ACKNOWLEDGMENTS}

This work was supported by grants from the National Key Project for Basic Research (2015CB150600), the National Natural Science Foundation of China (31200028 and 31471743), and the Natural Science Foundation of Guangdong Province (grant number 2014A030313455). The authors are grateful to J. E. Cronan at University of Illinois at Urbana-Champaign for his constructive revision of our manuscript.

\section{LITERATURE CITED}

Basconcillo, L. S., and McCarry, B. E. 2008. Comparison of three GC/MS methodologies for the analysis of fatty acids in Sinorhizobium meliloti: Development of a micro-scale, one-vial method. J. Chromatogr. B Analyt. Technol. Biomed. Life Sci. 871:22-31.

Campbell, J. W., and Cronan, J. E., Jr. 2001. Bacterial fatty acid biosynthesis: Targets for antibacterial drug discovery. Annu. Rev. Microbiol. 55: 305-332.

Debellé, F., Moulin, L., Mangin, B., Dénarié, J., and Boivin, C. 2001. Nod genes and Nod signals and the evolution of the Rhizobium legume symbiosis. Acta Biochim. Pol. 48:359-365.

Demont, N., Debellé, F., Aurelle, H., Dénarié, J., and Promé, J. C. 1993. Role of the Rhizobium meliloti nodF and nodE genes in the biosynthesis of lipo-oligosaccharidic nodulation factors. J. Biol. Chem. 268:20134-20142.

Demont-Caulet, N., Maillet, F., Tailler, D., Jacquinet, J. C., Promé, J. C., Nicolaou, K. C., Truchet, G., Beau, J. M., and Dénarié, J. 1999. Noduleinducing activity of synthetic Sinorhizobium meliloti nodulation factors and related lipo-chitooligosaccharides on alfalfa. Importance of the acyl chain structure. Plant Physiol. 120:83-92.

Feng, S.-X., Ma, J.-C., Yang, J., Hu, Z., Zhu, L., Bi, H. K., Sun, Y.-R., and Wang, H.-H. 2015. Ralstonia solanacearum fatty acid composition is determined by interaction of two 3-ketoacyl-acyl carrier protein reductases encoded on separate replicons. BMC Microbiol. 15:223.

Feng, S.-X., Zhu, L., Luo, B., Sun, Y.-R., and Wang, H.-H. 2008. Reconstitution of Escherichia coli fatty acid biosynthesis reaction in vitro. Prog. Biochem. Biophys. 35:954-963.

Fernandez-Aunión, C., Hamouda, T. B., Iglesias-Guerra, F., Argandoña, M., Reina-Bueno, M., Nieto, J. J., Aouani, M. E., and Vargas, C. 2010 Biosynthesis of compatible solutes in rhizobial strains isolated from Phaseolus vulgaris nodules in Tunisian fields. BMC Microbiol. 10:192.

Fontenelle, C., Blanco, C., Arrieta, M., Dufour, V., and Trautwetter, A 2011. Resistance to organic hydroperoxides requires $o h r$ and $o h r R$ genes in Sinorhizobium meliloti. BMC Microbiol. 11:100.

Galibert, F., Finan, T. M., Long, S. R., Puhler, A., Abola, P., Ampe, F., Barloy-Hubler, F., Barnett, M. J., Becker, A., Boistard, P., Bothe, G., Boutry, M., Bowser, L., Buhrmester, J., Cadieu, E., Capela, D., Chain, P., Cowie, A., Davis, R. W., Dreano, S., Federspiel, N. A., Fisher, R. F., Gloux, S., Godrie, T., Goffeau, A., Golding, B., Gouzy, J., Gurjal, M., Hernandez-Lucas, I., Hong, A., Huizar, L., Hyman, R. W., Jones, T., Kahn, D., Kahn, M. L., Kalman, S., Keating, D. H., Kiss, E., Komp, C., Lelaure, V., Masuy, D., Palm, C., Peck, M. C., Pohl, T. M., Portetelle, D., Purnelle, B., Ramsperger, U., Surzycki, R., Thebault, P., Vandenbol, M., Vorholter, F. J., Weidner, S., Wells, D. H., Wong, K., Yeh, K. C., and Batut, J. 2001. The composite genome of the legume symbiont Sinorhizobium meliloti. Science 293:668-672.

Gurvitz, A. 2009. The essential mycobacterial genes, fabG1 and fabG4, encode 3-oxoacyl-thioester reductases that are functional in yeast mitochondrial fatty acid synthase type 2 . Mol. Genet. Genomics 282: 407-416.

Horvath, B., Kondorosi, E., John, M., Schmidt, J., Török, I., Györgypal, Z., Barabas, I., Wieneke, U., Schell, J., and Kondorosi, A. 1986. Organization, structure and symbiotic function of Rhizobium meliloti nodulation genes determining host specificity for alfalfa. Cell 46:335-343.

Jacob, A. I., Adham, S. A., Capstick, D. S., Clark, S. R., Spence, T., and Charles, T. C. 2008. Mutational analysis of the Sinorhizobium meliloti short-chain dehydrogenase/reductase family reveals substantial contribution to symbiosis and catabolic diversity. Mol. Plant-Microbe Interact 21:979-987

Jiang, Y., Chan, C. H., and Cronan, J. E. 2006. The soluble acyl-acyl carrier protein synthetase of Vibrio harveyi B392 is a member of the medium chain acyl-CoA synthetase family. Biochemistry 45: 10008-10019.

Kavanagh, K. L., Jörnvall, H., Persson, B., and Oppermann, U. 2008. Medium- and short-chain dehydrogenase/reductase gene and protein families: The SDR superfamily: Functional and structural diversity within a family of metabolic and regulatory enzymes. Cell. Mol. Life Sci. 65:3895-3906. 
Khan, S. R., Gaines, J., Roop, R. M., 2nd, and Farrand, S. K. 2008. Broadhost-range expression vectors with tightly regulated promoters and their use to examine the influence of TraR and TraM expression on Ti plasmid quorum sensing. Appl. Environ. Microbiol. 74:5053-5062.

Lai, C. Y., and Cronan, J. E. 2004. Isolation and characterization of beta-ketoacyl-acyl carrier protein reductase $(f a b G)$ mutants of Escherichia coli and Salmonella enterica serovar Typhimurium. J. Bacteriol. 186:1869-1878.

López-Lara, I. M., and Geiger, O. 2001. The nodulation protein NodG shows the enzymatic activity of an 3-oxoacyl-acyl carrier protein reductase. Mol. Plant-Microbe Interact 14:349-357.

Maillet, F., Debellé, F., and Dénarié, J. 1990. Role of the nodD and syrM genes in the activation of the regulatory gene nodD3, and of the common and host-specific nod genes of Rhizobium meliloti. Mol. Microbiol. 4: 1975-1984.

Mao, Y. H., Ma, J. C., Li, F., Hu, Z., and Wang, H. H. 2015. Ralstonia solanacearum RSp0194 encodes a novel 3-keto-acyl carrier protein synthase III. PLoS One 10:e0136261.

Mergaert, P., Van Montagu, M., and Holsters, M. 1997. Molecular mechanisms of Nod factor diversity. Mol. Microbiol. 25:811-817.

Miller-Williams, M., Loewen, P. C., and Oresnik, I. J. 2006. Isolation of salt-sensitive mutants of Sinorhizobium meliloti strain Rm1021. Microbiology 152:2049-2059.

Oppermann, U., Filling, C., Hult, M., Shafqat, N., Wu, X., Lindh, M., Shafqat, J., Nordling, E., Kallberg, Y., Persson, B., and Jörnvall, H. 2003. Short-chain dehydrogenases/reductases (SDR): The 2002 update. Chem. Biol. Interact. 143-144:247-253.

Patankar, A. V., and González, J. E. 2009. An orphan LuxR homolog of Sinorhizobium meliloti affects stress adaptation and competition for nodulation. Appl. Environ. Microbiol. 75:946-955.

Rawlings, M., and Cronan, J. E., Jr. 1992. The gene encoding Escherichia coli acyl carrier protein lies within a cluster of fatty acid biosynthetic genes. J. Biol. Chem. 267:5751-5754.
Schäfer, A., Tauch, A., Jäger, W., Kalinowski, J., Thierbach, G., and Pühler, A. 1994. Small mobilizable multi-purpose cloning vectors derived from the Escherichia coli plasmids pK18 and pK19: Selection of defined deletions in the chromosome of Corynebacterium glutamicum. Gene 145:69-73.

Stover, C. K., Pham, X. Q., Erwin, A. L., Mizoguchi, S. D., Warrener, P., Hickey, M. J., Brinkman, F. S., Hufnagle, W. O., Kowalik, D. J., Lagrou, M., Garber, R. L., Goltry, L., Tolentino, E., Westbrock-Wadman, S., Yuan, Y., Brody, L. L., Coulter, S. N., Folger, K. R., Kas, A., Larbig, K., Lim, R., Smith, K., Spencer, D., Wong, G. K., Wu, Z., Paulsen, I. T., Reizer, J., Saier, M. H., Hancock, R. E., Lory, S., and Olson, M. V. 2000. Complete genome sequence of Pseudomonas aeruginosa PAO1, an opportunistic pathogen. Nature 406:959-964.

Swanson, J. A., Tu, J. K., Ogawa, J., Sanga, R., Fisher, R. F., and Long, S. R. 1987. Extended region of nodulation genes in Rhizobium meliloti 1021. I. Phenotypes of Tn5 insertion mutants. Genetics 117:181-189.

Ulrich, A. K., de Mendoza, D., Garwin, J. L., and Cronan, J. E., Jr. 1983. Genetic and biochemical analyses of Escherichia coli mutants altered in the temperature-dependent regulation of membrane lipid composition. J. Bacteriol. 154:221-230.

Wang, H., and Cronan, J. E. 2004. Only one of the two annotated Lactococcus lactis fabG genes encodes a functional beta-ketoacyl-acyl carrier protein reductase. Biochemistry 43:11782-11789.

Wang, L., Yang, J., Huang, C., and Wang, H. 2008. Overexpression and purification of Escherichia coli holo-acyl carrier protein and synthesis of acyl carrier protein. Acta Microbiol. Sin. 48:963-969.

White, S. W., Zheng, J., Zhang, Y. M., and Rock, C. O. 2005. The structural biology of type II fatty acid biosynthesis. Annu. Rev. Biochem. 74:791-831.

Zhang, Y. M., and Rock, C. O. 2008. Membrane lipid homeostasis in bacteria. Nat. Rev. Microbiol. 6:222-233.

Zhu, L., Lin, J., Ma, J., Cronan, J. E., and Wang, H. 2010. Triclosan resistance of Pseudomonas aeruginosa PAO1 is due to FabV, a triclosanresistant enoyl-acyl carrier protein reductase. Antimicrob. Agents Chemother. 54:689-698. 